\title{
Gyrokinetic study of ASDEX Upgrade inter-ELM pedestal profile evolution
}

\author{
D. R. Hatch \\ Institute for Fusion Studies, University of Texas at Austin, Austin, Texas 78712, USA
D. Told, H. Doerk, M. G. Dunne, E. Wolfrum, E. Viezzer, and The ASDEX Upgrade Team
Max-Planck-Institut für Plasmaphysik, Boltzmannstr. 2, D-85748 Garching, Germany \\ F. Jenko \\ Max-Planck-Institut für Plasmaphysik, Boltzmannstr. 2, D-85748 Garching, Germany and \\ Department of Physics and Astronomy, University of California, Los Angeles, California 90095, USA \\ M.J. Pueschel \\ University of Wisconsin-Madison, Madison, Wisconsin 53706, USA
}

\begin{abstract}
The gyrokinetic GENE code is used to study inter-ELM H-mode pedestal profile evolution for an ASDEX Upgrade discharge. Density gradient driven trapped electron modes (TEM) are the dominant pedestal instability during the early density-buildup phase. Nonlinear simulations produce particle transport levels consistent with experimental expectations. Later inter-ELM phases appear to be simultaneously constrained by electron temperature gradient (ETG) and kinetic ballooning mode (KBM) turbulence. The electron temperature gradient achieves a critical value early in the ELM cycle, concurrent with the appearance of both microtearing modes (MTM) and ETG modes. Nonlinear ETG simulations demonstrate that the profiles lie at a nonlinear critical gradient. The nominal profiles are stable to KBM, but moderate increases in $\beta$ are sufficient to surpass the KBM threshold. Certain aspects of the dynamics support the premise of KBM-constrained pedestal evolution; the density and temperature profiles separately undergo large changes, but in a manner that keeps the pressure profile constant and near the KBM limit.
\end{abstract}

\section{INTRODUCTION}

The H-mode [1] is an improved tokamak confinement regime characterized by an edge transport barrier with steep temperature and density gradients. H-mode operation with a sufficiently large pedestal top temperature (in the range of $\sim 4$ $\mathrm{keV}$ ) is thought to be necessary in order for ITER to achieve its fusion power targets [2]. This imperative is challenged by the activity of edge localized modes (ELMs) [3] — cyclical collapses of the edge pedestal, which, on an ITER-scale machine, would release unacceptable heat loads on plasma facing components. ITER operation must achieve the fine balance of producing a robust pedestal while avoiding large ELMs.

The stability of magnetohydrodynamic (MHD) modes typically defines upper bounds on what pedestal parameters are achievable preceding an ELM crash [4, 5]. ELM-free scenarios [6-12] rely on some other transport mechanism to arrest pedestal evolution before the ELM-triggering MHD limits are reached. In this context, a critical question is how pedestal density and temperature profiles evolve in response to heat and particle transport fluxes and their respective sources. One might ask on the one hand whether such transport mechanisms may be sufficient to avoid ELMs, and on the other hand whether they might preclude realization of the $4 \mathrm{keV}$ ITER pedestal target. For example, various oscillations-sometimes quasi-coherent-are observed in pedestals that apparently affect transport and ELMS. Thus it is desirable to develop an understanding of pedestal profile evolution that goes beyond the basics of the bounding MHD limits. In this work we explore this question by using gyrokinetic simulations in conjunction with time-resolved inter-ELM pedestal profiles to probe the interaction between pedestal profile evolution and kinetic microinstabilities.

Various transport mechanisms have been proposed for the pedestal [13, 14], including neoclassical [15], paleoclassical [16, 17], and microturbulent transport. Several analyses of pedestal microinstabilities and profile evolution have been published in recent years [18-21]. In Ref. [21], local linear gyrokinetic simulations were used to study the inter-ELM behavior of MAST pedestal profiles. A transition from microtearing modes (MTM) to kinetic ballooning modes (KBM) was observed as the pedestal recovered. In Ref. [19], local gyrokinetic simulations were applied to DIII-D pedestal profiles. MTM was observed at the pedestal top and drift waves were identified in the pedestal. KBM was found to be subdominant but weakly unstable. In Ref. [18, 20], global gyrokinetic simulations were used to study DIII-D pedestal profiles and found KBM unstable in the pedestal and a kinetic peeling ballooning mode unstable prior to the ELM crash.

Here we use the GENE code [22] to study the relation between pedestal instabilities and profile evolution during several inter-ELM time periods of an ASDEX Upgrade (AUG) discharge. In this paper we report on local flux-tube results. The fully developed pedestal width corresponds to approximately fifty gyroradii. For such scale lengths a local treatment is likely to capture at least qualitatively most of the salient dynamics. Nonetheless, global operation is certainly preferable and is the focus of ongoing work. Nonlinear simulations of trapped electron mode (TEM) and electron temperature gradient (ETG) driven turbulence are used to characterize the transport associated with these instabilities and elucidate various properties of the turbulence. Future work will focus on global effects and nonlinear KBM simulations. 
In this work we study three inter-ELM time periods from an AUG H-mode discharge similar to those described in Refs. [23, 24]. The profile evolution is characterized by an early density-buildup phase during which TEM dominates, and later phases during which the profile evolution appears to be simultaneously constrained by ETG and KBM turbulence.

The early density-buildup phase occurs $\sim 3 \mathrm{~ms}$ after the ELM crash. Density gradient driven TEM is predominant during this phase and is characterized by significant particle transport (as quantified by the ratio of normalized particle to heat flux). Nonlinear simulations are used to characterize the TEM particle transport.

The second phase of interest is an intermediate phase $\sim 8$ ms after the ELM crash during which the profiles are still evolving, but the electron temperature gradient has reached a critical value and the outer pedestal is near the KBM limit. Nonlinear ETG simulations demonstrate that the profiles are at a nonlinear critical gradient; heat fluxes increase by an order of magnitude with moderate adjustments to background gradients.

The third phase of interest represents the final pre-ELM state whose characteristics are largely maintained for the final half of the ELM cycle. During this phase the outer pedestal remains near the KBM limit. The profile evolution between the second and third stages is consistent with a KBM-constrained pedestal; the density and temperature profiles evolve separately in a manner that maintains a near-constant pressure profile over the outer half of the pedestal.

This paper is outlined as follows. In Sec. II the ASDEX Upgrade discharge is described, along with the available experimental data. In Sec. III we describe certain technical details of the gyrokinetic GENE simulations performed, the properties of the microinstabilities identified, and the procedure used for identifying ballooning limits. In Sec. IV we examine in detail the relation between the observed microinstabilies and the profile evolution during each phase and characterize the TEM and ETG transport. In Sec. V the details of the nonlinear simulations are described. A summary and discussion are provided in Sec. VI.

\section{EXPERIMENTAL DETAILS}

The discharge chosen for this analysis was AUG discharge \#27963, which featured a 1 MA plasma current, a $-2.5 \mathrm{~T}$ field and medium average triangularity of 0.25 in a lower single null configuration. A mixture of neutral beam injection and electron cyclotron resonance heating were used in this discharge. Timetraces of (a) heating power, (b) deuterium fueling rate, (c) normalised plasma beta, (d) core (black) and edge (blue) line integrated densities, and (e) ELM frequency are shown in Fig. 1. In order to increase the radial resolution of the diagnostics, a radial sweep of the plasma position by $2 \mathrm{~cm}$ was performed between 4 and $4.5 \mathrm{~s}$. Data for the profiles used in this work were taken between 3 and $4.5 \mathrm{~s}$ where all plasma parameters (apart from the radial position) and the ELM frequency were constant. This particular shot is well suited to the analysis undertaken here due to the long inter-ELM time of $30 \mathrm{~ms}$.

The electron temperature and density profiles were interpreted by using the electron cyclotron forward modelling (ECFM) method [25]. This accurately models the measured radiation temperature from electron cyclotron emission in the plasma edge and determines the correct electron temperature based on this. The electron density profiles are also fitted as part of this interpretation. The edge ion temperatures were measured using poloidal and toroidal charge exchange recombination spectroscopy diagnostics which also allows the radial electric field in the pedestal to be calculated [26] and aligned with the other kinetic profiles.

The data from the analysed time range were combined into a single composite ELM cycle, as discussed in Ref. [24], allowing a highly temporally resolved analysis of the ELM recovery. Several time windows during the ELM cycle, shown in Fig. 2 were chosen for further analysis. The data during each of these time windows were fitted to provide constraints for the magnetic equilibrium, and also for the GENE simulations. From the measured kinetic profiles and the extensive set of poloidal magnetic field sensors installed at AUG, an accurate equilibrium was also calculated using the CLISTE code [27, 28] for each time point. Previous work on AUG [29] has shown that this allows the edge current density, and hence magnetic shear profile, to be determined with a high degree of accuracy.

The ELM period in shot 27963 is approximately $30 \mathrm{~ms}$ and is characterized by a complex succession of several distinct phases. The time-resolved maximum pedestal temperature and density gradients are shown in Fig. $2(t=0$ represents the ELM crash). Magnetic equilibria and temperature and density profiles have been reconstructed for six inter-ELM time points (represented by the shaded regions in Fig. 2). Each reconstruction is averaged over $\sim 2 \mathrm{~ms}$ and separated by $\sim 5$ ms. Additional details can be found in Refs. [23, 24]. In the following analysis, we focus largely on the segments at $t=3.5,7.5,-2.5 \mathrm{~ms}$ with respect to the ELM crash, which will be denoted S3, S4, and S1, respectively, consistent with the labels in Fig. 2. Several important features of the profiles are shown in Fig. 3: the electron density profiles (Fig. 3 A), the electron temperature profiles (Fig. $3 \mathrm{~B}$ ), the normalized electron density gradient scale lengths $a / L_{n}$ (Fig. $3 \mathrm{C}$ ), the normalized electron temperature gradient scale lengths $a / L_{T e}$ (Fig. 3 D), the ratio of the scale lengths $\eta_{e}=L_{n e} / L_{T e}$ (Fig. 3 $\mathrm{E}$ ), and the pressure (ion plus electron) profiles (Fig. $3 \mathrm{~F}$ ). The radial coordinate $\rho_{t o r}=\left(\Psi_{\varphi} / \Psi_{\varphi 0}\right)^{1 / 2}$ is the square root of the normalized toroidal magnetic flux. The ion profiles are also experimentally diagnosed and are qualitatively similar to the electron profiles. The major distinction is in the temperature profiles; whereas the electron temperature gradient increases early in the ELM cycle and remains constant thereafter (see Fig. 3 D), the ion temperature profile remains flat until later in the ELM cycle.

Phases S3 and S4 are characterized by rapidly evolving profiles (note the large differences in the density and temperature profiles shown in Fig. $3 \mathrm{~A}$ and $\mathrm{B}$, respectively). While the density and temperature profiles evolve in a complex fashion, the resulting pressure profile evolution follows a simple 
trend: the pressure profile rises and steepens, approaching its final pre-ELM value at radial points that move progressively inward with time (see Fig. 3 F). After $\sim 13.5 \mathrm{~ms}$, the profiles have largely recovered their final pre-ELM characteristics, and only comparatively minor variations are observed over the remaining time points. We study the final pre-ELM segment $\mathrm{S} 1(-2.5 \mathrm{~ms})$ in detail.

The radial electric field $\left(E_{r}\right)$ profiles $[26,30]$ and the resulting $E \times B$ shear rates [31] are also highly relevant. The force balance that determines $E_{r}$ is largely governed by the main ion pressure gradient [26], so that the $E_{r}$ profile recovers in tandem with the pressure profile. The $E_{r}$ profiles for $\mathrm{S} 3$ and $\mathrm{S} 1$ are shown in Fig. $4 \mathrm{~A}$, and the $E \times B$ shear rates [32]

$$
\Omega_{E \times B}=\frac{B_{\theta}^{2} R^{2}}{B} \frac{d}{d \psi} \frac{E_{r}}{B_{\theta} R}
$$

are shown in Fig. 4 B. In Eq. 1, $\psi$ denotes the poloidal flux, $B_{\theta}$ is the outboard midplane poloidal magnetic field, and $\mathrm{R}$ is the outboard midplane major radius. The shear rates greatly exceed the maximum low- $k_{y}$ growth rates throughout the pedestal in S1, where $k_{y}$ is the wave number corresponding to the binormal $y$ coordinate. In S3, there is a portion of the pedestal centered around $\rho_{\text {tor }}=0.95$ where the shear rate is small, allowing low- $k_{y}$ TEM turbulence to be active. Although the $E_{r}$ profile for $\mathrm{S} 4$ is not available, the similarity of the S4 and S1 pressure profiles indicates that large shear rates have likely been reestablished.

Note that the Hahm-Burrell shear rate defined above is different from the shear rate used as the input parameter for GENE, which is defined as

$$
\Omega_{E \times B}=\frac{\rho_{\text {tor }}}{q} \frac{d}{d \rho_{\text {tor }}} \Omega_{\text {tor }},
$$

where $q$ is the safety factor and $\Omega_{\text {tor }}$ is the toroidal rotation. This definition produces values that are nearly an order of magnitude smaller than the corresponding Hahm-Burrell shear rates $\left(\Omega_{\mathrm{tor}}=E_{r} / B_{\theta} R\right.$ is used in this calculation). The GENE shear rates are also plotted in Fig. 4 (solid lines) in order to emphasize that in the pedestal different definitions of shear rates can significantly affect interpretation of standard comparisons between shear rates and growth rates.

\section{GYROKINETIC ANALYSIS}

\section{A. Description of simulations}

The GENE code [22] is a comprehensive gyrokinetic code that includes electromagnetic effects (including $B_{\|}$), a linearized Landau-Boltzmann collision operator, and an interface with experimental equilibria and profiles, all of which are used routinely in this study. GENE is used largely in its local linear mode, with targeted nonlinear simulations used to verify and explore certain conclusions. Global simulations and more extensive nonlinear simulations will be the focus of future work.

As will be described below, a wide range of microinstabilities is manifest in the AUG pedestal. These require varying

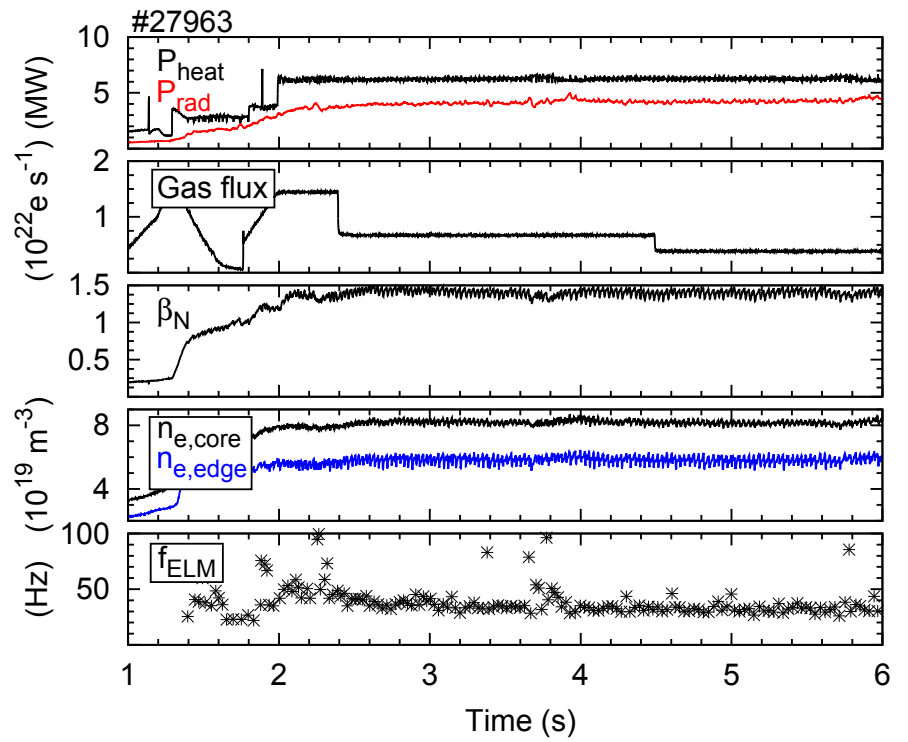

FIG. 1: Time traces of (a) heating power, (b) deuterium fueling rate, (c) normalised plasma beta, (d) core (black) and edge (blue) line integrated densities, and (e) ELM frequency for AUG discharge \#27963.

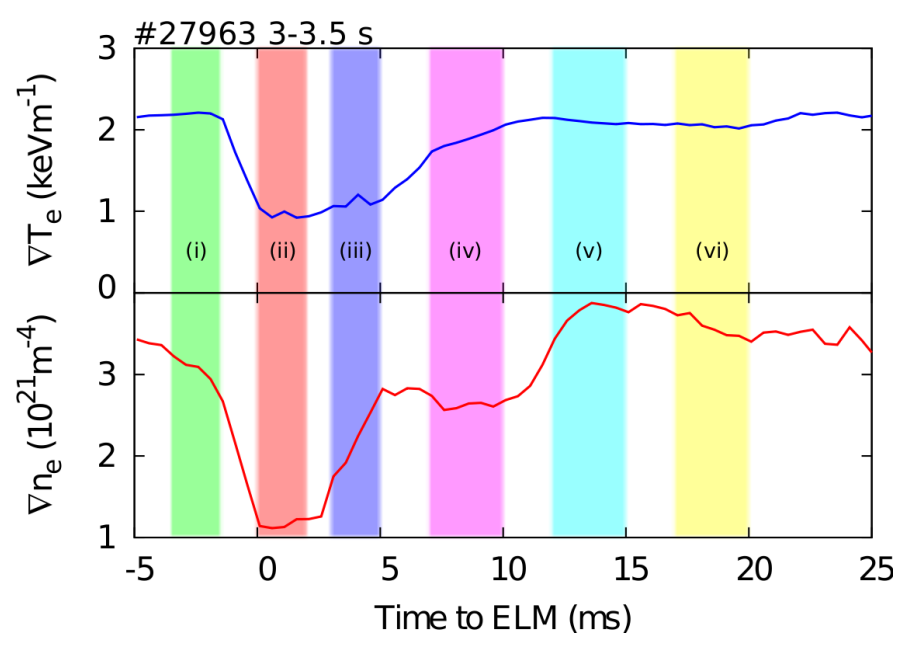

FIG. 2: Time-resolved maximum pedestal temperature (top) and density (bottom) gradients for ASDEX Upgrade shot 27963. The shaded regions denote the segments for which profiles and magnetic equilibria have been reconstructed. Segments S3 (iii), S4 (iv), and S1 (i) are studied in detail.

amounts of grid resolution to properly resolve. The highdimensional parameter space of interest (spanning radial location, binormal wavenumber, inter-ELM time point, and various scans in gradient scale lengths and $\beta$ ) precludes exhaustive convergence tests for every linear simulation. In the course of this study, convergence tests were performed for cases representative of the major microinstabilities in each time phase. Moreover, important mode characteristics, like ballooning mode structures for the electrostatic potential $\phi$ and the parallel magnetic vector potential $A_{\|}$were consistently monitored in order to avoid any indication of numerical under-resolution. We routinely used $128-192$ grid points 

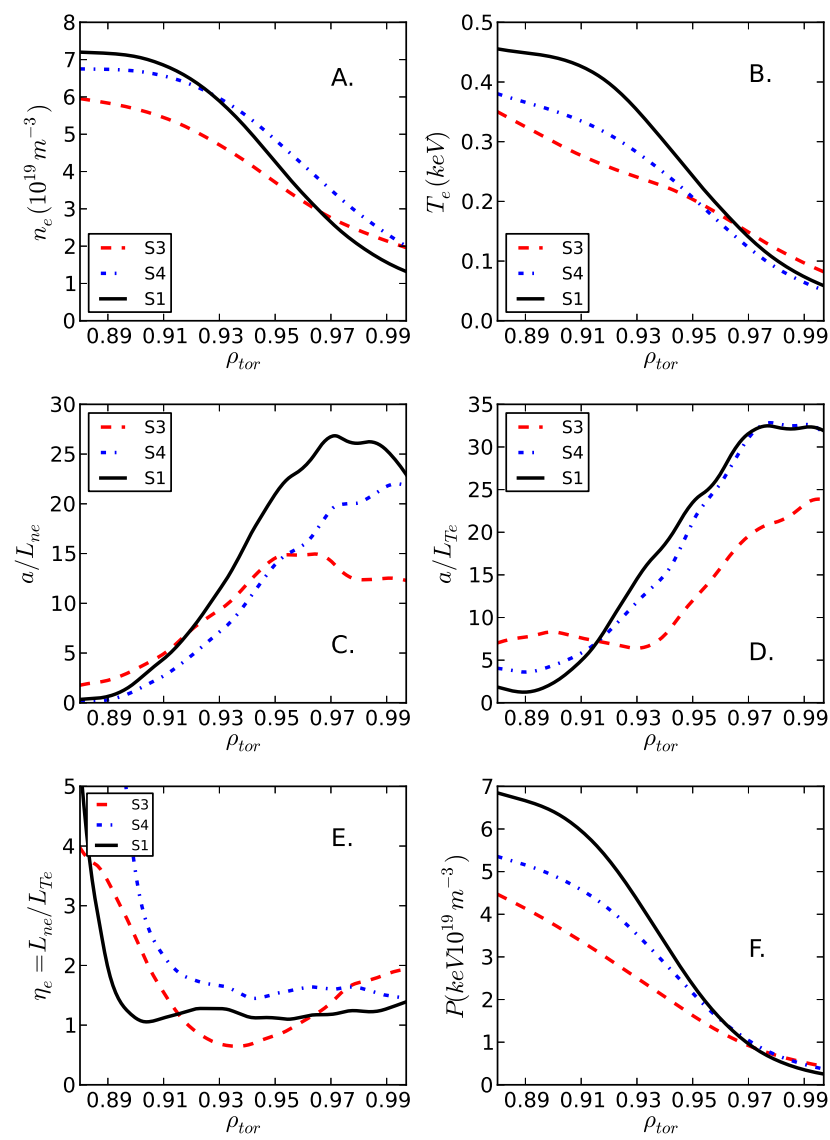

FIG. 3: Various features of the profiles during S3 ( density buildup phase-dashed, red online), S4 (intermediate phase-dot-dashed, blue online), and S1 (final pre-ELM phase-solid, black). The electron density profiles are shown in A, the electron temperature profiles in B, the normalized inverse density gradient scale length $a / L_{n e}$ in $\mathrm{C}$, the normalized inverse electron temperature gradient scale length $a / L_{T e}$ in $\mathrm{D}$, the ratio of the scale lengths $L_{n e} / L_{T e}$ in $\mathrm{E}$, and the pressure (ion plus electron) profiles.

in the parallel coordinate $z, 64$ grid points in parallel velocity space $v_{\|}, 16$ grid points for the magnetic moment $\mu$, and $5-9$ $k_{x}$ (where $k_{x}$ is the wave number corresponding to the radial coordinate $x$ ) modes linked by the flux tube parallel boundary condition [33]. This resolution was chosen to resolve the most difficult modes in the high-dimensional parameter space, and thus typically over-resolves the majority of the microinstabilities simulated. Fourth-order hyperdiffusion [34] was used in the parallel coordinate in order to stabilize grid-scale numerical modes. Numerical details regarding the global simulations and nonlinear simulations will be discussed in the relevant sections.
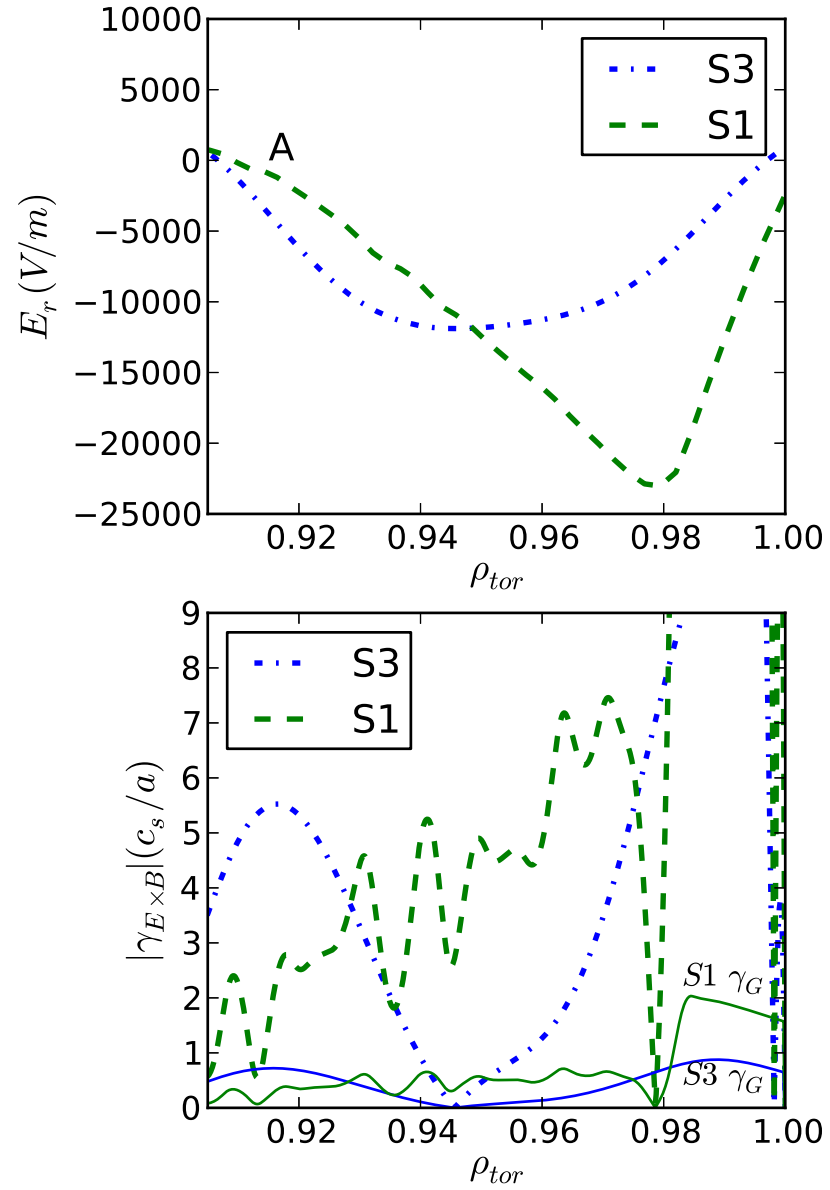

FIG. 4: A. The radial electric field $E_{r}$ profiles for S3 (dot-dashed line, blue online), and S1 (dashed line, green online). B. The HahmBurrell $E \times B$ shear rates for S3 (dot-dashed line, blue online) and S1 (dashed line, green online). The shear rates calculated using the definition used for GENE input are also shown (solid lines, labelled $\left.\gamma_{G}\right)$.

\section{B. Important microinstabilities}

Linear gyrokinetic GENE simulations identify four important microinstabilities in the different phases of the AUG interELM pedestal evolution: density-gradient-driven TEM, ETG modes, MTM, and KBM.

TEM [35-37] instabilities are observed at intermediate $k_{y}$ scales during all inter-ELM phases, and are (almost) the sole microinstability observed during S3 ( $\beta$ and pressure gradients are too low to excite KBM, and $\eta_{e}$ is too small to excite electron modes during S3). The TEM growth rates typically increase with $a / L_{n}$ and decrease with increasing $a / L_{T e}$. The mode is characterized by mode structures that extend to larger ballooning angles (higher $k_{x}$ ) than the other modes, and the amplitude often peaks away from the outboard midplane 
$(z=0)$ (a feature also observed in Refs. [38, 39]). Ref. [38] notes that density gradient driven TEM develops this doublepeaked mode structure at sufficiently high density gradients. The mode structures of the electrostatic potential $\phi$ and the parallel magnetic vector potential $A_{\|}$are shown in Fig. 5 A and $\mathrm{B}$. The dispersion relation for the TEM exhibits a mode frequency that often transitions from negative to positive frequencies as $k_{y}$ increases, as can be seen in Fig. 8 .

MTMs are small-scale, electron-temperature-gradientdriven analogs to MHD tearing modes [40] that produce (almost exclusively) electron thermal transport. They are unstable at low $k_{y}$ during $\mathrm{S} 4$ and $\mathrm{S} 1$, and have negative frequencies that strongly increase with $k_{y}$. MTMs have often been observed to be unstable inside the pedestal top [19,21,41], as is also observed in this AUG discharge. A representative mode structure is shown in Figs. 5 C and D. Note that the up-down asymmetry of the magnetic equilibrium allows the modes to deviate from the exact symmetry about the outboard midplane $(z=0)$ that would otherwise be enforced.

KBM shares many of the same properties of its counterpart, the ideal ballooning, including being driven (primarily) by pressure gradients (i.e., they are driven by contributions from gradients in temperature and density for all species). Representative mode structures are shown in Figs. 5 E and F. KBM produces robust particle and thermal transport and constrains pedestal pressure gradients in many [5, 14] (but possibly not all $[14,42]) \mathrm{H}$-mode scenarios. Some experimental observations are also consistent with KBM properties [43, 44].

The properties of ETG modes include instability at small scales $\left(k_{y} \rho_{i} \gg 1\right)$, predominantly electron thermal transport, a threshold in $\eta_{e}=L_{n e} / L_{T e}$, and negative frequencies that increase with $k_{y}[45,46]$. ETG modes are unstable at small scales during all phases later than S3. Analysis of ETG instability in AUG pedestals is also discussed in Refs. [47-49].

\section{Discussion of Ballooning Limits}

The proximity of the pedestal profiles to the KBM and ideal ballooning limits is of particular interest. Predictions of pedestal properties appeal to KBM instability in setting the scaling of the pedestal width with $\beta$ [50], thereby providing a constraint on the pedestal height. Here we discuss a procedure for identifying the linear ballooning limits (which appear to be a good proxy for nonlinear limits [51]) in the context of the modes observed in these simulations. We first note that (as will be described in the following sections) all nominal pedestal profiles are stable to KBM. However, KBM can be destabilized by increasing $\beta$ (or alternatively gradients) to various degrees.

Two transitions to the critical $\beta$ KBM limit are observed. First, a sharp transition from MTM to KBM similar to that described in [21]. Second, a smooth transition is observed from a mode with an electron-diamagnetic (negative) frequency and a more-complex mode structure to a KBM, which is characterized by a smoother more strongly-ballooning mode structure and an ion-diamagnetic (positive) frequency. This transition is illustrated in Fig. 6, which demonstrates that the mode
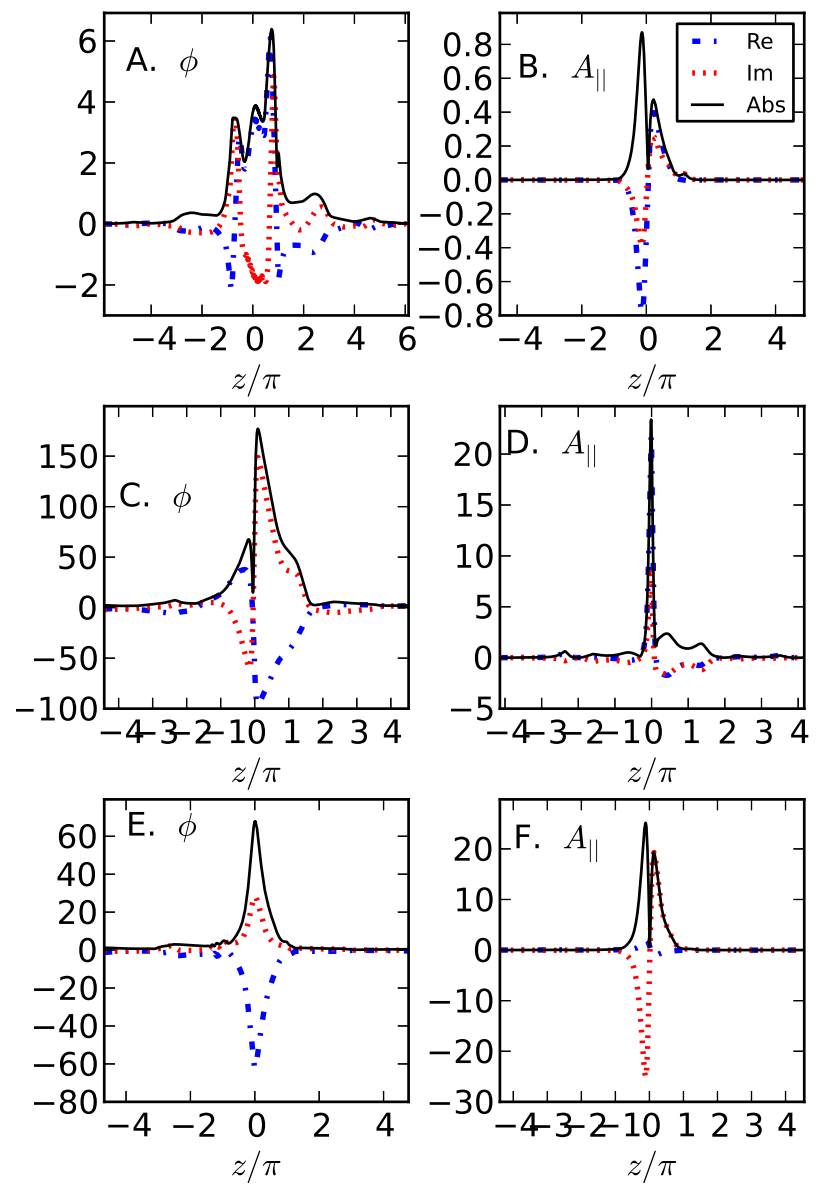

FIG. 5: Representative parallel mode structures for three important micro-instabilities: the electrostatic potential $\phi$ and the parallel magnetic vector potential $A_{\|}$for a TEM (A and B), a microtearing mode ( $\mathrm{C}$ and $\mathrm{D})$, and a kinetic ballooning mode ( $\mathrm{E}$ and $\mathrm{F})$.

structures become smoother and more peaked as $\beta$ increases, and Fig. 7, which shows the dispersion relation as $\beta$ increases from its nominal value to a value that is $30 \%$ greater. In Fig. 7, the MTM-KBM transition is manifest at $k_{y} \rho_{s}=0.04,0.05$, and the smooth transition is observed at higher wave numbers. Note that the growth rates in the range $k_{y} \rho_{s} \sim 0.1-0.16$ exhibit very little sensitivity to the initial increase in $\beta$, indicating that the electromagnetic nature of the mode undergoes a fundamental change as it transitions to ion diamagnetic frequency. The mode structures in Fig. 6 correspond to $k_{y} \rho_{s}=0.08$ in Fig. 7. We define the KBM limit as the value at which the modes transition to ion-diamagnetic frequencies, which in this case corresponds to $\beta=1.1 \times \beta_{0}$.

The KBM limit is reached at a lower value than the ideal ballooning limit [51, 52]. Since the KBM threshold converges to that of infinite-n ideal ballooning modes in the limit $k_{y} \rightarrow$ 0 [53], we can estimate the ideal ballooning limit as the $\beta$ 


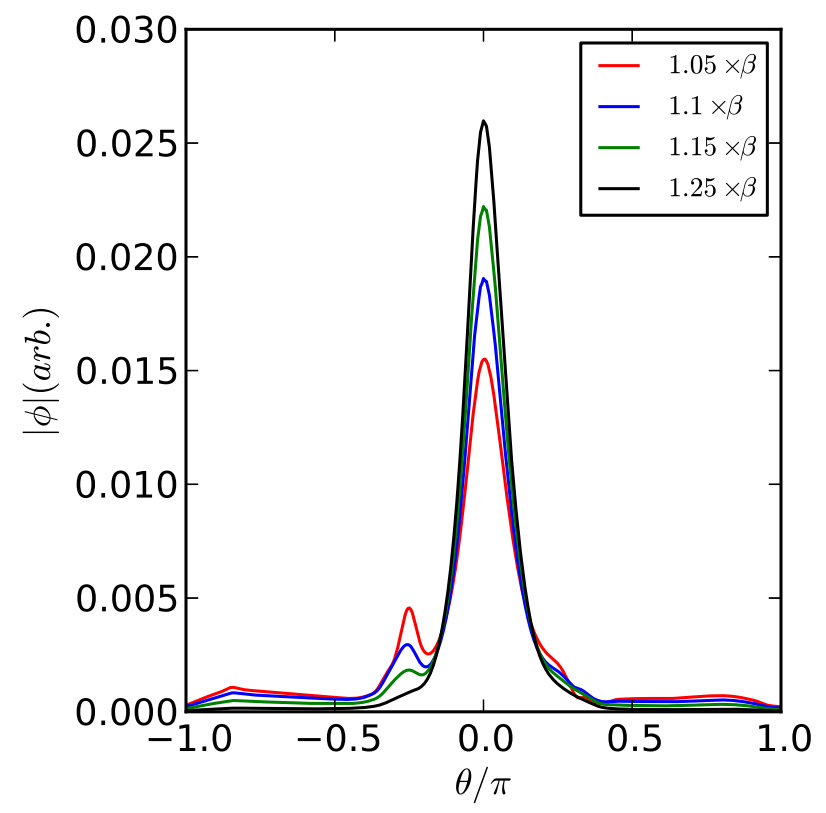

FIG. 6: Parallel mode structures of the electrostatic potential as the mode transitions from a TEM to a KBM. The mode structure becomes smoother and more peaked as $\beta$ increases. This scan corresponds to $k_{y} \rho_{s}=0.08$ in Fig. 7

value at which an extrapolation of the growth rates to $k_{y}=0$ is positive. This occurs at roughly $\beta=1.15 \times \beta_{0}$ in Fig. 7 . This procedure will be used to characterize the proximity of the profiles to ballooning instability in the next sections.

\section{INTER-ELM PHASES}

In this section we examine each phase of the profile evolution in detail with the goal of characterizing transport and identifying connections between the profile evolution and the underlying microinstabilities.

\section{A. Phase S3}

During S3, TEM is the dominant instability across the pedestal, as seen in Fig. 8, which shows growth rates and frequencies for various radial locations. The $E_{r}$ profile has not fully recovered during this phase, leaving a significant window of low $E \times B$ flow shear in the region centered at $\rho_{\text {tor }}=0.95$, as seen in Fig. 4. Thus, we may expect low- $k_{y}$ turbulence to be active in this region, which is consistent with the profile evolution, as described below. In order to interpret the profile dynamics during S3, we consider the variation from $\mathrm{S} 3$ to $\mathrm{S} 4$ to provide a rough time derivative for the quantities of interest (compare the red and blue lines in Fig. 3). During this phase, the density across the entire pedestal rises sharply, as seen in Fig. 3 A. A key question is the mechanism for this

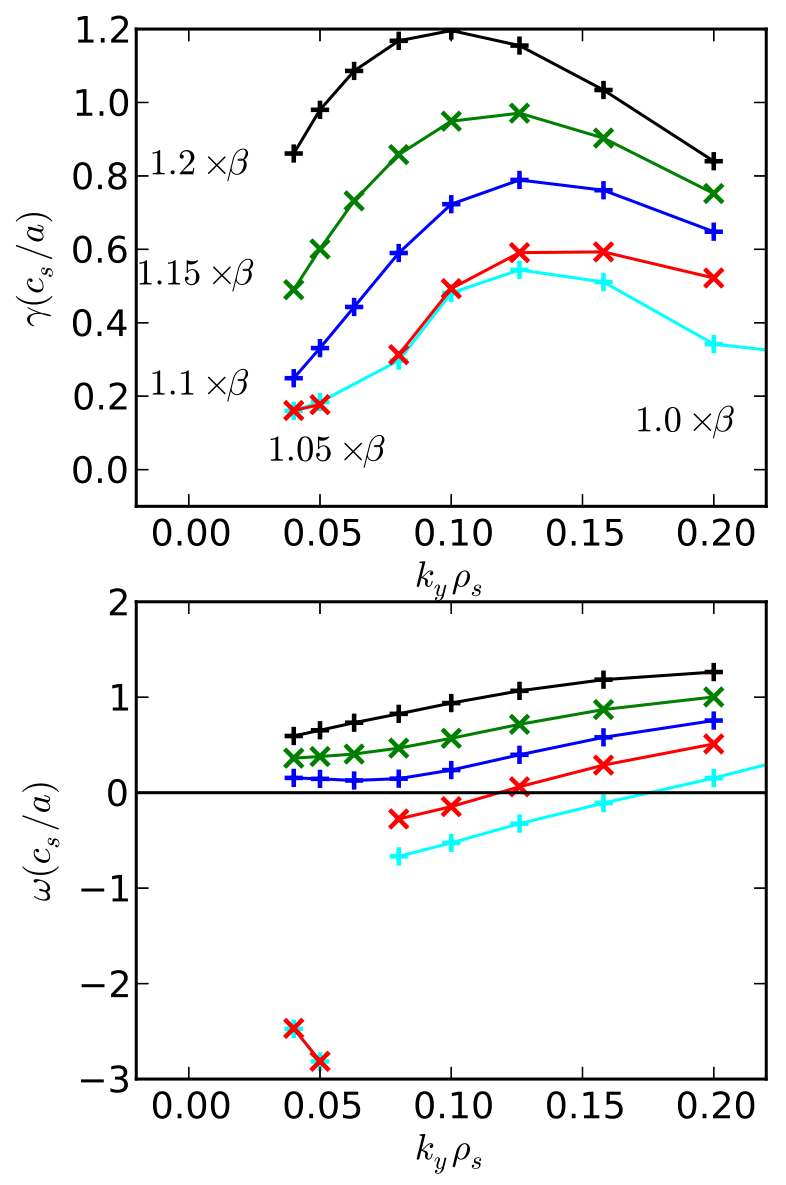

FIG. 7: Growth rates (top) and frequencies (bottom) for a $\beta$ scan demonstrating the transition to $\mathrm{KBM}$ instability. At $k_{y} \rho_{s}=$ $0.04,0.05$, there is a transition from MTM to KBM. At higher $k_{y}$, the mode smoothly transitions from a TEM whose growth rate is insensitive to $\beta$ to a KBM whose growth rate strongly increases with $\beta$.

density buildup. The two candidate mechanisms [13, 54-56] are neutral fueling and a particle pinch, the latter mechanism being beneficial for ITER, which will have a narrow neutral penetration length [57].

A nonlinear TEM simulation produced a particle flux $\Gamma / \Gamma_{G B}=6.8 \pm 1.1$, with $\Gamma_{G B}=n_{0} c_{s} \rho_{s}^{2} / a^{2}$ (for reference, the neoclassical contribution to the particle flux was calculated by GENE to be $\left.\Gamma_{N C} / \Gamma_{G B}=1.54\right)$. This net outward transport can be reconciled with the density buildup by appealing to a particle source that dominates the transport. The TEM particle flux translates into a diffusivity $D=0.025 \pm 0.004 \mathrm{~m}^{2} / \mathrm{s}$, which is very similar to the quantity $\left(D=0.031 \mathrm{~m}^{2} / \mathrm{s}\right)$ determined by predictive-iterative modeling to best fit the profile evolution of the density buildup during the L-H transition of a similar AUG discharge [55]. A scan of the density gradient scale length was conducted in order to extract diffusive and pinch components of the flux. However simulations with 


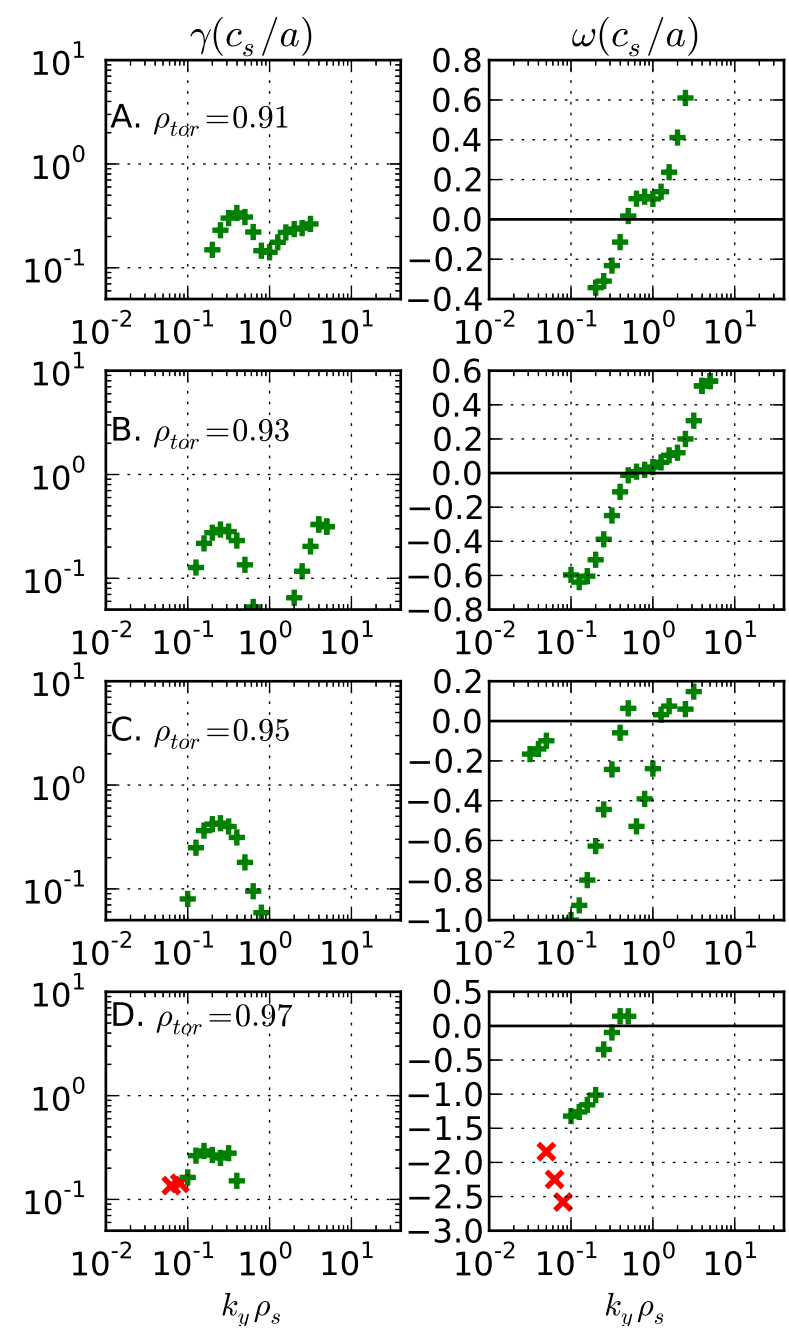

FIG. 8: Growth rates and frequencies for S3 at $\rho_{\text {tor }}=0.91$ (A), $\rho_{\text {tor }}=0.93(\mathrm{~B}), \rho_{\text {tor }}=0.95(\mathrm{C}), \rho_{\text {tor }}=0.97$ (D). TEM are denoted by + (green online) and microtearing modes are denoted by $\mathrm{x}$ (red online).

$15 \%$ and $30 \%$ increases in $a / L_{n}$ produced widely different estimates of diffusive and pinch components.

This analysis indicates that the particle source (i.e., neutral fueling) is the dominant mechanism for the density buildup in this scenario. However, the possibility of a substantial TEMdriven particle pinch in other pedestal scenarios cannot be ruled out.

\section{B. Phase $\mathbf{S 4}$}

S4 is characterized (in relation to S3) by much steeper temperature gradients but comparable density gradients over much of the pedestal. Thus $\eta_{e}$ is large enough to excite electron instabilities like MTM at low $k_{y}$ and ETG at high $k_{y}$, as seen in Fig. 10. As in S3, TEM (green) is still unstable at intermediate scales. Although the $E_{r}$ data is not available during $\mathrm{S} 4$, the recovering pressure profile (whose gradient determines $E_{r}$ ) indicates that substantial shear rates are to be expected during this phase, likely stabilizing the intermediatescale TEM. The effect of shear-flow on MTMs is still an open question, with some results indicating an insusceptibility of MTMs to shear flow [58], and others indicating strong suppression due to shear flow [59].

During this phase, the temperature gradient scale lengths have already approached a critical value across the pedestal that is maintained through the remainder of the ELM cycle (compare the gradients for S4 and S1 in Fig. 3 D). This is in spite of the fact that the temperature itself is still increasing over much of the pedestal as seen in Fig. 3 B. The fixed temperature gradient may be due to either the low- $k_{y}$ MTM or the high- $k_{y}$ ETG modes, both of whose growth rates increase strongly with the temperature gradient.

Nonlinear MTM simulations and multi-scale ETG simulations are beyond the scope of this work. Nonlinear electron scale ETG simulations (described in more detail below in Sec. V) indicate that the profiles are at a nonlinear critical gradient-moderate variations in gradient scale lengths (increasing $a / L_{T e}$ by $20 \%$ and decreasing $a / L_{n}$ by $20 \%$ ) are sufficient to increase the heat flux by an order of magnitude, as shown in Fig. 9.

The resulting power flowing through the flux surfaces of interest ranges from tens to hundreds of $\mathrm{kW}$ depending on the gradient modifications as shown in Fig. 9 B. This discharge is powered by $5 \mathrm{MW}$ of neutral beam injection (NBI) heating and $1 \mathrm{MW}$ of electron cyclotron resonance heating (ECRH). Radiation accounts for 3-5 MW of power loss, leaving 1-3 MW attributable to transport mechanisms. The resulting electron power flows may also be modified to various degrees by ohmic heating, and collisional and turbulent energy exchange with ions. Further ambiguity is introduced by the dynamic nature of the pedestal evolution. In light of the uncertainties, a conservative conclusion is that ETG is plausibly responsible for a significant fraction of the electron heat flux in the pedestal of this discharge, but other transport mechanisms are likely necessary to account for the full transport level. The drastic increase in ETG transport with moderate gradient variations suggests that the electron temperature gradient is constrained by ETG turbulence. Candidate mechanisms for additional electron heat transport include neoclassical, and MTM and KBM turbulence. Paleoclassical transport has also been proposed as a pedestal transport mechanism [13].

The profiles during S4 are already quite close to the KBM limit in the outer region of the pedestal. This is consistent with the pressure profiles shown in Fig. $3 \mathrm{~F}$, which are almost identical during phases $\mathrm{S} 4$ and $\mathrm{S} 1$ at positions $\rho_{\text {tor }} \gtrsim 0.95$. Increases in $\beta$ of $10 \%$ and $15 \%$ are sufficient to excite KBM at $\rho_{\text {tor }}=0.95$ and $\rho_{\text {tor }}=0.97$, respectively (also shown with black symbols in Fig. 10). Variations of $\beta$ of this magnitude are justified by experimental uncertainties in the profiles, which range from $\sim 10 \%$ to $\sim 30 \%$ across the pedestal (note that, e.g., a $15 \%$ increase in $\beta$ can be achieved with only $\sim 7 \%$ increases in both the temperature and density).

KBM activity during S4 is also supported by the observation that the density decreases during the transition between 

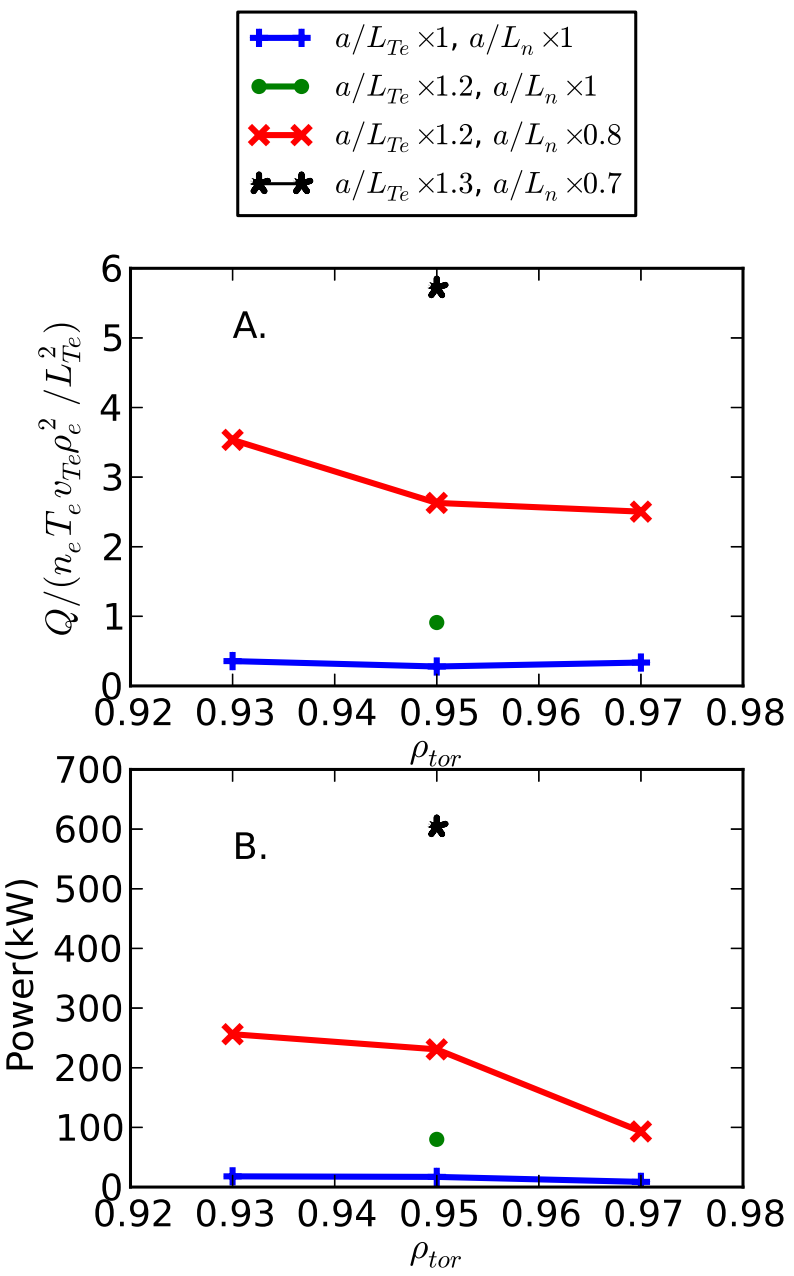

FIG. 9: Electron heat flux (A) and the corresponding power transported through flux surfaces (B) attributable to ETG turbulence using variations of the background gradients as input. The sharp increase in transport with changes in gradients is an indication that the profiles lie near a nonlinear critical gradient for ETG turbulence. The transport can reach experimentally significant levels with moderate modifications to the gradients.

S4 and S1. The region of decreasing density $\left(\rho_{\text {tor }} \gtrsim 0.95\right)$ corresponds closely to the region where the profiles are near the KBM limit. The temperature in this region increases concurrent with this decrease in density, suggesting that density and temperature profiles are free to evolve as long as the pressure profile is kept at the KBM limit.

\section{Phase S1}

The profiles achieve their final pre-ELM characteristics approximately $13 \mathrm{~ms}$ after the ELM crash, so that phases S5, S6, and $\mathrm{S} 1$ have largely similar properties with respect to microinstabilities. We analyze S1 in detail. Similar to S4, MTMs are unstable at low- $k_{y}$ and ETG modes are unstable at high $k_{y}$ as seen in Fig. 11. The TEM has become almost completely sta-
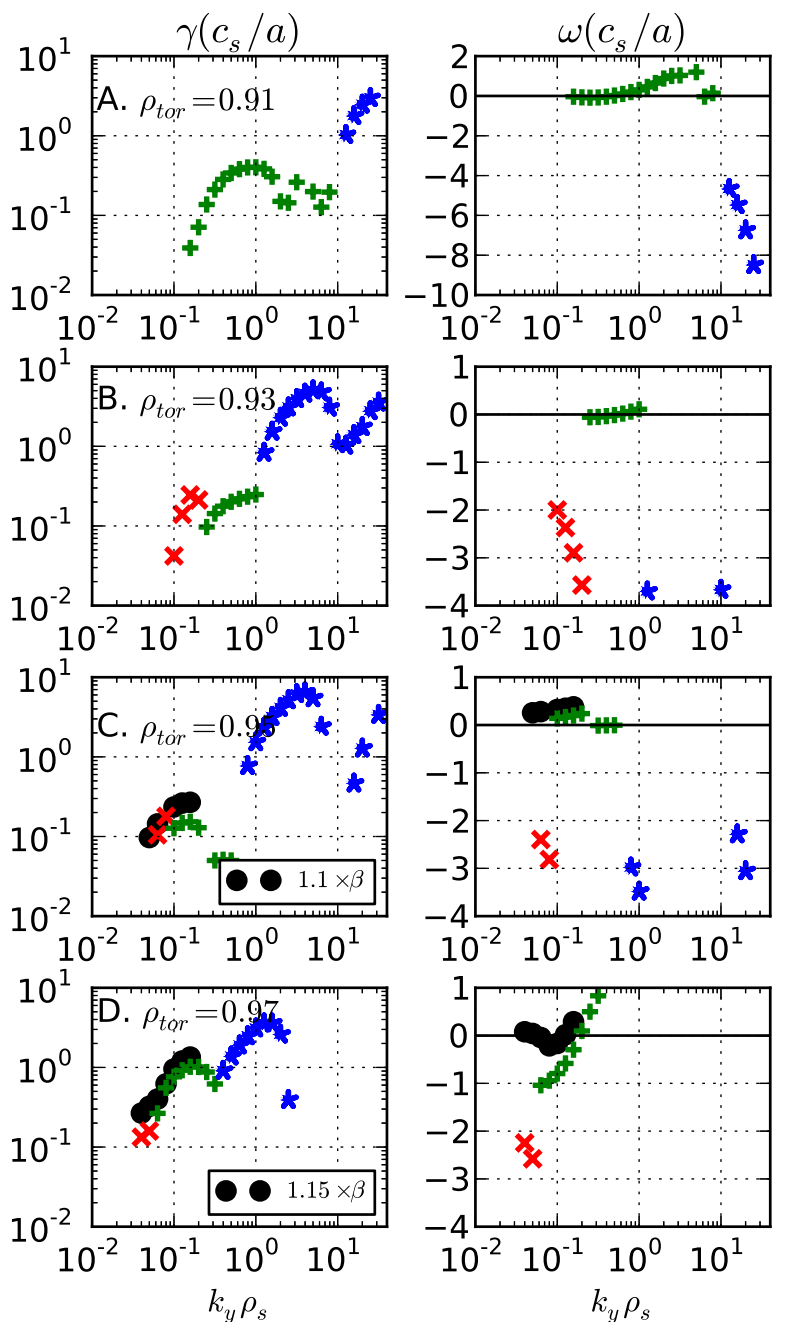

FIG. 10: Growth rates and frequencies for S4 at $\rho_{\text {tor }}=0.91$ (A), $\rho_{\text {tor }}=0.93(\mathrm{~B}), \rho_{\text {tor }}=0.95(\mathrm{C}), \rho_{\text {tor }}=0.97(\mathrm{D})$. TEM is denoted by + (green online), MTM is denoted by x (red online), and ETG modes are denoted by asterisks (blue online). KBM growth rates and frequencies, attained by increasing $\beta$ by 20 and $10 \%$ are shown in $\mathrm{C}$ and $\mathrm{D}$, respectively, and are denoted by circles. Note that the frequency plot range is kept small, eliminating many ETG frequencies, in order to make the ion-scale dispersion relations discernible.

bilized at $\rho_{\text {tor }}=0.93,0.95$. Similar to $\mathrm{S} 4$, scans in $\beta$ show that the nominal profiles in the outer pedestal lie just below both the KBM and ideal ballooning $\beta$ limits. At $\rho_{\text {tor }}=0.97$, the KBM limit is reached with a $10 \%$ increase in $\beta$, while the infinite-n ideal ballooning limit is reached with a $15 \%$ increase (as already discussed in subsection III C and shown in Fig. 7). At $\rho_{\text {tor }}=0.95$ and $\rho_{\text {tor }}=0.93$ the KBM limit is reached with increases of $20 \%$ and $30 \%$, respectively. In these cases there appears to be little separation between the infinite-n and KBM limits. The $30 \% \beta$ increase necessary to find the KBM limit at $\rho_{\text {tor }}=0.93$ is easily justified when uncertainties in gradients (in addition to $\beta$ ) are also taken into account.

The premise of KBM-constrained profile evolution is 

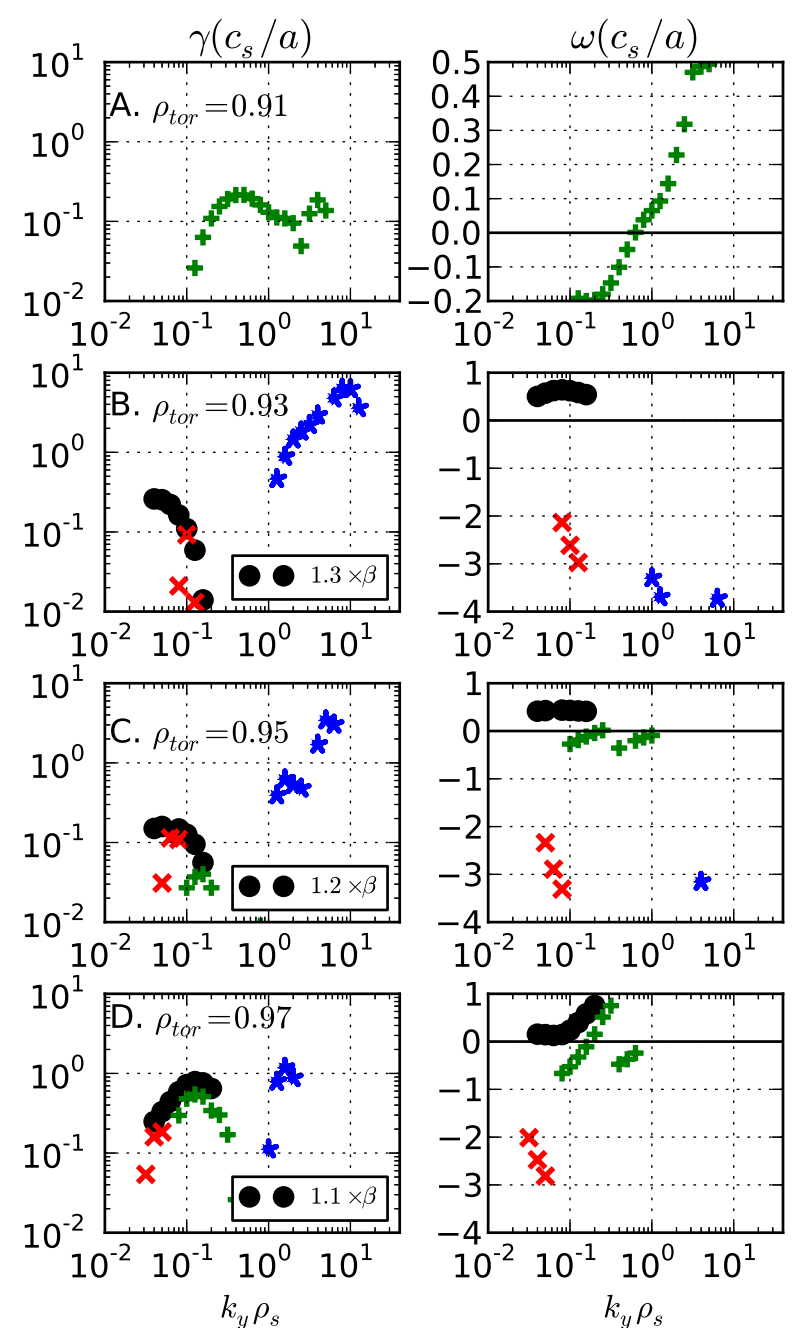

FIG. 11: Growth rates and frequencies for $\mathrm{S} 1$ at $\rho_{\text {tor }}=0.91$ (A), $\rho_{\text {tor }}=0.93(\mathrm{~B}), \rho_{\text {tor }}=0.95(\mathrm{C}), \rho_{\text {tor }}=0.97$ (D). TEM is denoted by + (green online), MTM is denoted by $\mathrm{x}$ (red online), and ETG modes are denoted by asterisks (blue online). KBM growth rates and frequencies, attained by increasing $\beta$ by 30,20 , and $10 \%$ are shown in B, C, and D, respectively, and are denoted by circles. Note that the frequency plot range is kept small, eliminating many ETG frequencies, in order to make the ion-scale dispersion relations discernible.

strengthened by the manner in which the density and temperature profiles evolve during the transition from S4 to S1. In the region $\rho_{\text {tor }}>0.95$, the density decreases and the temperature increases in such a way that the pressure profile itself is unchanged (compare Figs. 3 A [density], B [electron temperature], and $\mathrm{F}$ [ion plus electron pressure]). Note that the ion temperature (not shown) increases more than the electron temperature. This is consistent with the observation that the profiles during each segment are of comparable proximity to the KBM limit, each requiring increases in $\beta$ of approximately 10 to $20 \%$.

\section{PREDICTED TURBULENCE PROPERTIES}

In this section we describe the numerical details and physical properties of nonlinear simulations of TEM turbulence during S3 and ETG turbulence during S4.

\section{A. Properties of TEM Turbulence}

Nonlinear simulations were performed for the S3 case centered at $\rho_{\text {tor }}=0.95$. During this phase, the flow shear is very weak in this radial region, allowing the TEM turbulence to be active. We use GENE in a local mode with Dirichlet boundary conditions enforcing zero fluctuations at the edge of the box. Local simulations with periodic radial boundary conditions saturate only with very large box sizes (several times the pedestal width) and at extremely high transport levels. Fully global nonlinear simulations are beyond the scope of this work and will be left for future studies. The effect of retaining the global shear flow profile (implemented as toroidal rotation with a rotation frequency $\Omega=E_{r} /\left(R B_{\theta}\right)$ across the radial simulation domain was tested and resulted in only minor quantitative differences with the shear-free simulations. A radial box size of $48 \rho_{s}$ was used (corresponding to $\left.\rho_{\text {tor }}=(0.92-0.98)\right)$, with $8 \%$ of the radial domain on each side devoted to buffer zones for enforcing the Dirichlet boundary condition. Resolution of $(96,96,64,32)$ grid points in the $\left(x, z, v_{\|}, \mu\right)$ directions was used. A box size of $125 \rho_{s}$ in the $y$ direction was used, which for $32 k_{y}$ modes corresponds to $k_{y, \min } \rho_{s}=0.05$ and $k_{y, \max } \rho_{s}=1.55$. Various convergence tests were conducted, including doubling the $x$ resolution, doubling the $y$ box size for fixed $k_{y, \max }$, and increasing $k_{y \text {,max }}$ by a factor of 1.5 for fixed box size. Spectra exhibit power-law decay and are shown in Fig. 12 for the electrostatic potential.

A distinctive feature of the TEM is the peaking of the transport away from the outboard midplane [38]. This is observed in the linear eigenmodes, and is retained in the nonlinear turbulence as seen in Fig. 14, which shows the parallel dependence of the fluxes peaking at $z=-0.75 \pi$ (near the inboard midplane) for both the linear and nonlinear scenarios. Other linear features of the turbulence are also maintained in the nonlinear system, albeit with substantial modification. The cross-phase angle $\alpha=-i \ln \left(\phi_{k}^{*} n_{k} /\left|\phi_{k}^{*} n_{k}\right|\right)$ in the nonlinear simulations has the same qualitative dependence on $k_{y}$ as the linear phase angle, but is shifted significantly toward $\alpha=0$. This comparison is shown in Fig. 13, which shows a time-accumulated probability distribution function (PDF) of the nonlinear cross phase angle along with the corresponding linear phase angles; the nonlinear phase angles are consistently down-shifted from the linear phase angles. The shift is most pronounced in the range $k_{y} \rho_{s}=0.2-0.4$, where the most unstable mode appears to be different from the neighboring (in $k_{y}$ ) modes, and a subdominant mode with comparable growth rate is likely the most active mode in the turbulence. Even for the lower wavenumbers where the spectrum peaks $\left(k_{y}=0.1\right)$, the shift is larger than is seen in many core-like turbulence systems $[36,60]$, and may be an indication of sub- 

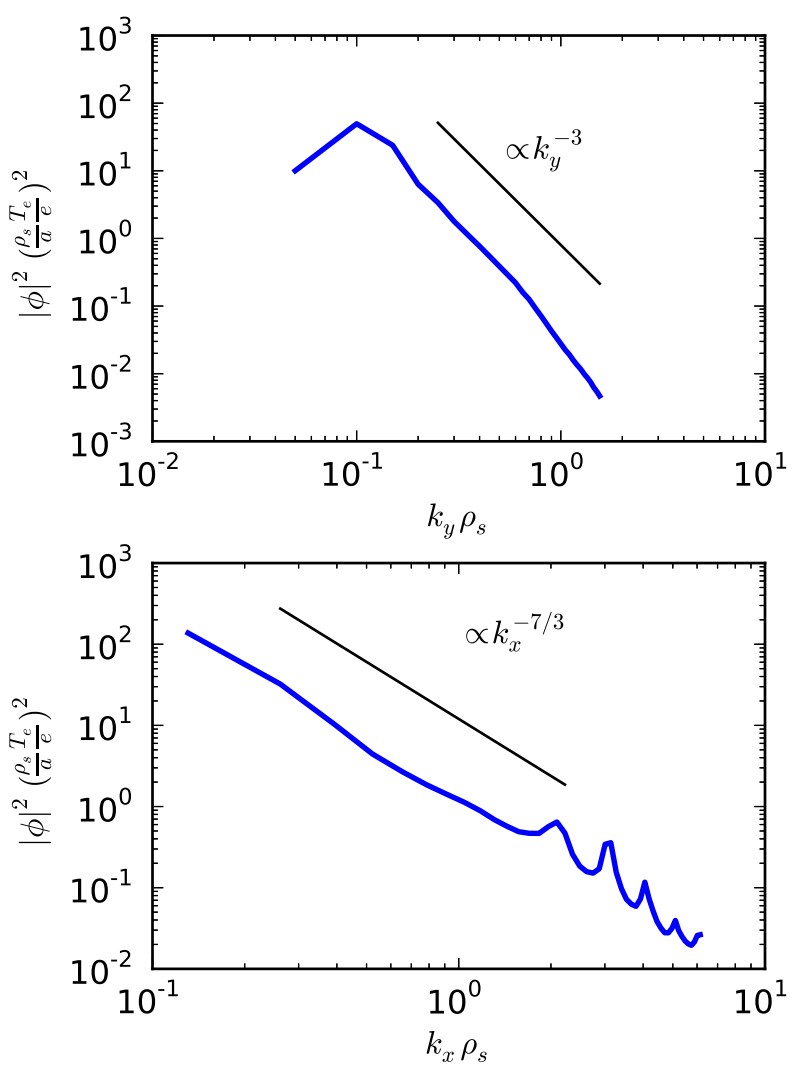

FIG. 12: Spectra ( $k_{y}$ summed over $k_{x}$, top, and $k_{x}$ summed over $k_{y}$, bottom) for the electrostatic potential for a simulation of TEM turbulence during S3. The bumps at high $k_{x}$ are physical and can be attributed to the flux-tube parallel boundary condition.

stantial damped eigenmode excitation [61-63]. Quasilinear models [64] would need to account for such a strong nonlinear modification of the cross phase in order to accurately model such transport. Significant deviations from linear properties for edge drift wave turbulence are also described in Ref. [65].

Zonal flows [66] are very active in the dynamics, with a large ratio of the electrostatic potential intensity at zonal wavenumbers to nonzonal wavenumbers: $\left|\phi_{k_{y}=0}\right|^{2} /\left|\phi_{k_{y} \neq 0}\right|^{2}=6$. The zonal-flow-regulated nature of the turbulence would suggest that such turbulence may, under certain conditions, be subject to the so-called non-zonal transition [67]-enhanced transport associated with degradation of zonal flows by magnetic fluctuations. Proximity to the nonzonal transition (as described in Refs. [67-69]) was tested for this simulation and the turbulence was found to be far from any such threshold, likely due to low fluctuation levels.

\section{B. Properties of ETG Turbulence}

Nonlinear ETG simulations were performed for the S4 phase at $\rho_{\text {tor }}=0.93,0.95,0.97$ using the adiabatic ion ap-

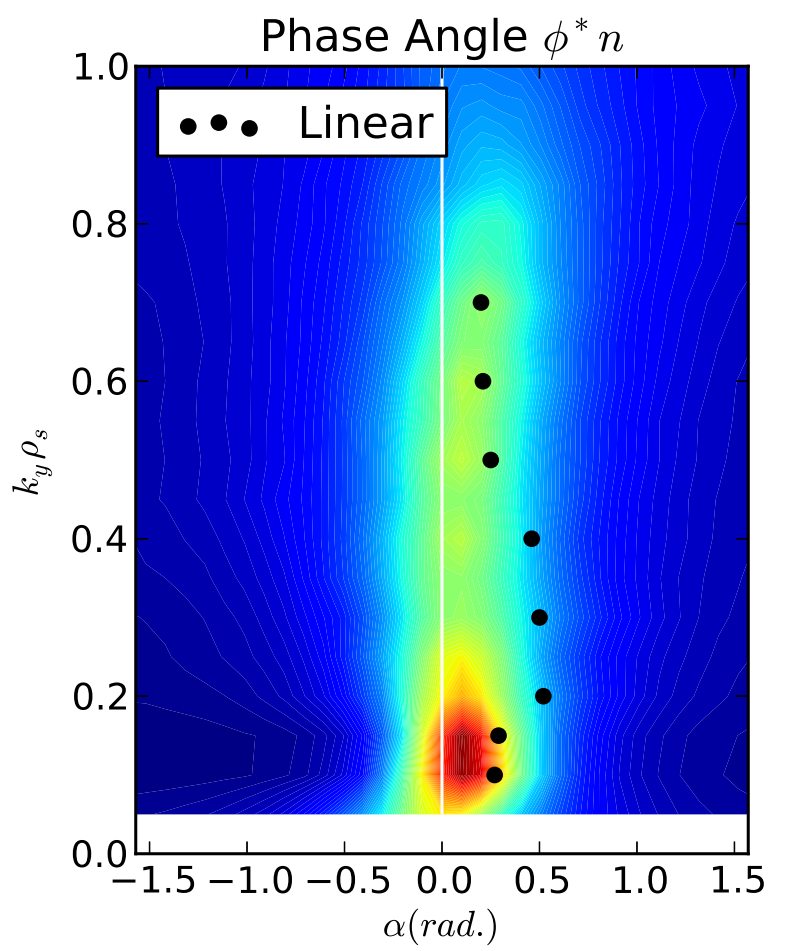

FIG. 13: Comparison of linear (black dots) and nonlinear (contours) $\phi * n$ cross phases for TEM turbulence during S3. Note the nonlinear reduction of the cross phase.

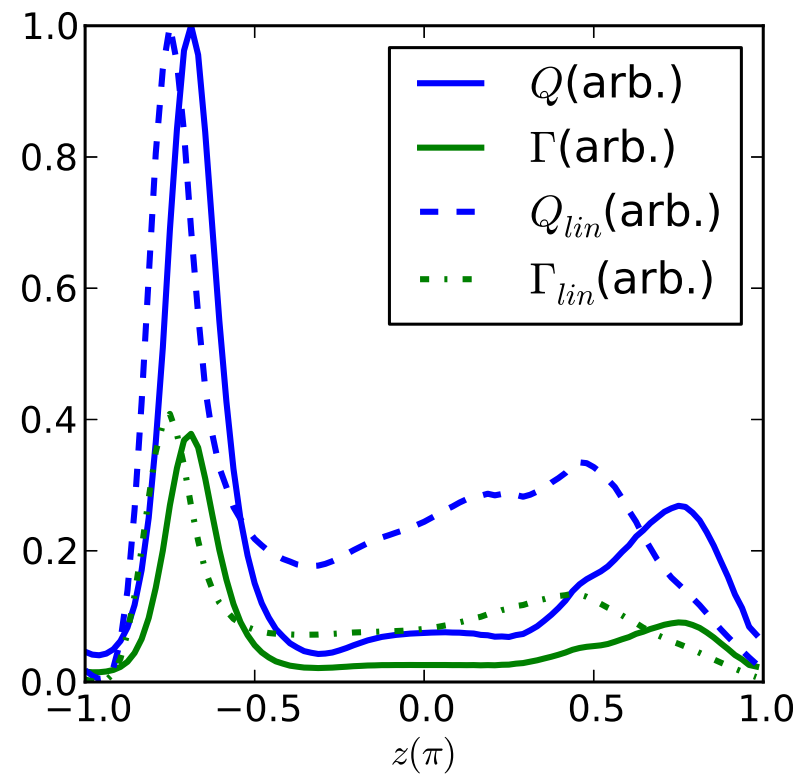

FIG. 14: Parallel dependence of the heat and particle fluxes for the nonlinear case (solid lines) and linear case (at $k_{y} \rho_{s}=0.1$, dashed and dot-dashed lines). The fluxes for both cases peak near the inboard side. 
proximation. A radial box size of $3.1 \rho_{s}$ was used in conjunction with $192 k_{x}$ modes (positive and negative). The minimum $y$ wavenumber for the simulations was $k_{y} \rho_{s}=5.0$ in conjunction with 64 modes (positive) resolving up to $k_{y} \rho_{s}=315$. Convergence in the perpendicular coordinates was established with extensive convergence tests. For the remaining phase space, resolution of $(78,32,10)$ grid points in the $\left(z, v_{\|}, \mu\right)$ coordinates was used.

The nominal gradients produce small transport levels and flux spectra with significant contribution from the low- $k_{y}$ portion of the spectrum, indicating that the single-scale treatment is inadequate for these cases. Increasing $a / L_{T e}$ by $20 \%$ produces an increase by a factor of five in the heat flux and shifts the transport spectrum to predominantly ETG scales, indicating that the single-scale treatment becomes viable at slightly higher gradients. Further modification of the gradients $\left(a / L_{T e}\right.$ increased by $20 \%$ and $a / L_{n}$ decreased by $\left.20 \%\right)$ results in an order of magnitude increase in transport. These results are summarized in Fig. 9. Gradient adjustments to this degree are justifiable in light of the 10 to $30 \%$ uncertainties in the experimental profiles, resulting in even larger uncertainties for the gradients. Note that the gyroBohm normalization used in Fig. $9 \mathrm{~A} Q_{G B}=n_{e} T_{e} v_{T e} \rho_{e}^{2} / L_{T e}^{2}$, which uses the electron temperature gradient scale length and electron values for the gyroradius and thermal velocity, is well suited to the resulting transport; the fluxes are of order unity and vary only moderately over the different radial locations (even though $v_{t e}, \rho_{e}$, and $a / L_{T e}$ vary enormously over the same range).

The high sensitivity of the ETG heat flux to modifications of the gradients slightly away from the nominal values is an indication that the profiles lie at a nonlinear critical gradient for ETG. Since ETG growth rates depend on $\eta_{e}=L_{n} / L_{T e}$, this would suggest that high density gradients are a precondition for the accessibility of high electron temperature gradients. Since both $a / L_{n}$ and $a / L_{T e}$ contribute to KBM drive, the increase in both gradients would ultimately be constrained by the KBM stability limit.

The heat flux spectrum for the $\rho_{t o r}=0.95$ case with the $20 \%$ modification to both temperature and density gradients is shown in Fig. 15; the flux peaks at $k_{y} \rho_{s} \sim 40$, and almost all the transport occurs at scales larger than $k_{y} \rho_{s} \sim 150$. Contours of the electrostatic potential are shown in Fig. 16, which accounts for the appropriate geometric coefficients in order to show both coordinates according to physical scale. Similar to ETG turbulence described in [49], the turbulence is largely isotropic, exhibiting no pronounced streamers or zonal flows.

\section{SUMMARY AND DISCUSSION}

We have presented a gyrokinetic analysis of time resolved ASDEX Upgrade inter-ELM pedestal profile evolution. The pedestal evolution entails an early density-buildup phase during which density gradient driven TEM is the predominant instability, and later phases that are likely constrained by both ETG and KBM turbulence.

The early density-buildup phase $(\sim 3.5 \mathrm{~ms}$ into the 30 ms ELM cycle) produces almost exclusively TEM instability.

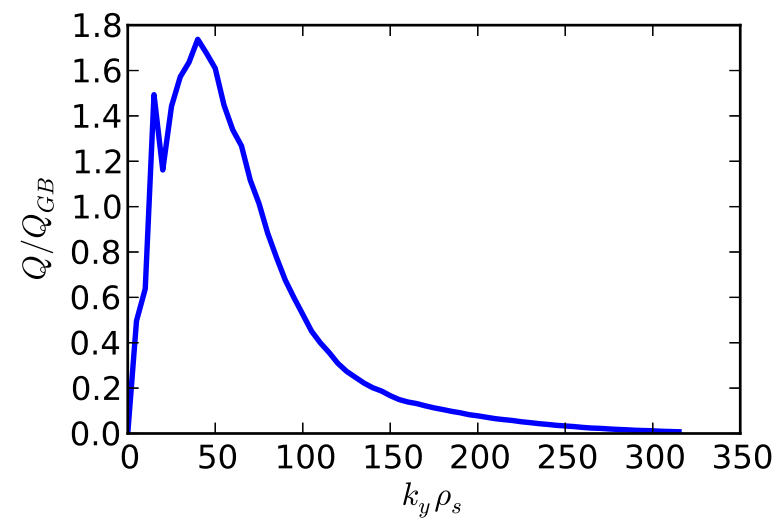

FIG. 15: The $k_{y}$ spectrum (summed over $k_{x}$ ) of the electron heat flux from a nonlinear ETG simulation using values of $a / L_{T e}$ and $a / L_{n}$ that were modified by $20 \%$.

The $E \times B$ flow shear is weak across much of the pedestal during this phase, allowing low- $k_{y}$ TEM turbulence to be active. Nonlinear simulations produce transport levels that are consistent with experimental expectations; the diffusivity $D=0.025 \mathrm{~m}^{2} / \mathrm{s}$ calculated by GENE is very close to the value calculated in an analysis of the L-H transition in a similar ASDEX Upgrade discharge [55].

The electron temperature gradients appear to reach a critical value early in the ELM cycle (at $\sim 8.5 \mathrm{~ms}$ ) concurrent with the appearance of both MTM and ETG instability. The ETG transport was characterized with nonlinear single-scale ETG simulations. The nominal gradients produce low levels of heat flux, but the transport exhibits a high degree of stiffness, increasing by orders of magnitude with reasonable variations of the temperature and density gradients. These results suggest that ETG turbulence is plausibly responsible for a significant portion of the electron heat flux, but other mechanisms are likely necessary to account for the full transport level. MTM and KBM turbulence are candidates for producing additional electron heat flux.

The nominal profiles lie just below the KBM limit in the outer half of the pedestal during the last two-thirds of the ELM cycle. Increases in $\beta$ of $10 \%$ to $20 \%$ (within the experimental error bars) are sufficient to excite KBM. Two factors support the premise that KBM constrains the pedestal evolution. First, after the initial density buildup, the density profile decreases across the outer pedestal at the same time and radial locations at which the profiles approach the KBM limit. Second, the density and temperature profiles separately undergo significant changes in the outer pedestal, but in a manner that keeps the pressure profile fixed and near the KBM limit.

These results suggest the possibility that the pedestal is simultaneously constrained by both ETG and KBM turbulence. The ETG constraint would mandate an increasing density gradient prior to or concurrent with an increasing electron temperature gradient in order to prevent $\eta_{e}$ from becoming too large. The KBM constraint would ultimately arrest the increase of both gradients since both gradients contribute to 


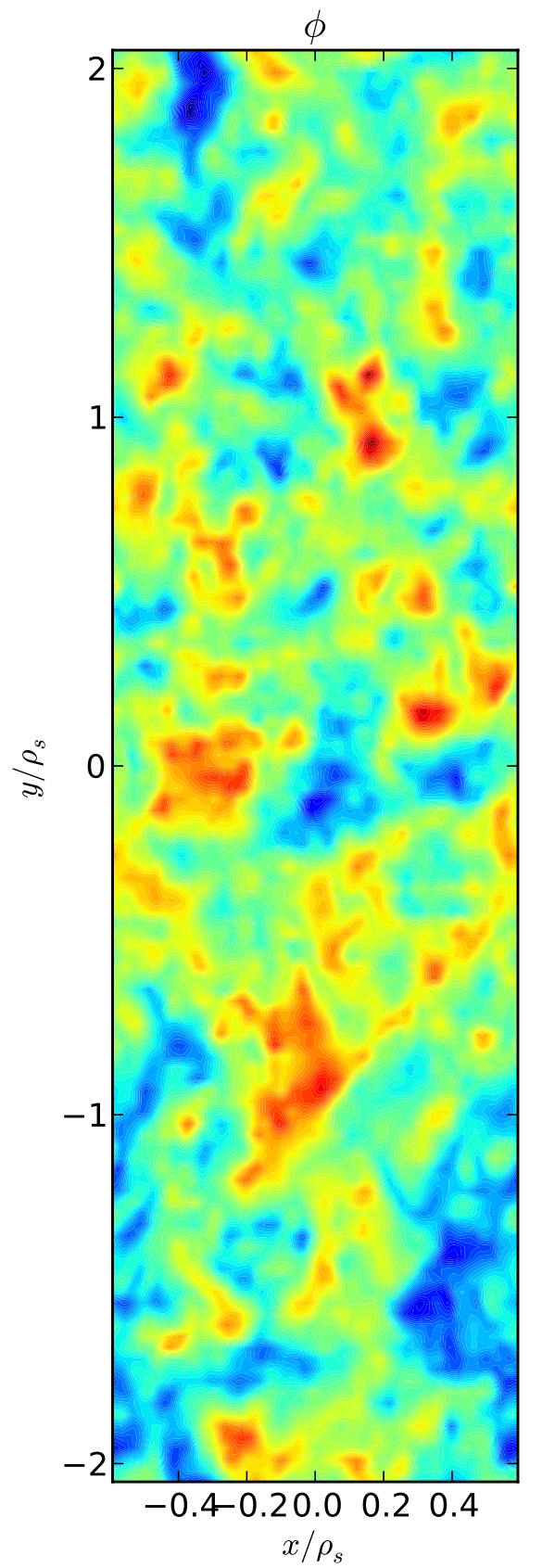

FIG. 16: Contour plots of the fluctuating electrostatic potential from an ETG simulation. The $\mathrm{x}$ and $\mathrm{y}$ axes are plotted to scale (including geometric corrections), demonstrating the absence of streamers.
KBM drive.

This work elucidates several ways in which turbulent transport can constrain pedestal evolution, providing a foundation for the study of additional effects (e.g., global effects and nonlinear KBM turbulence) in the pedestal and exploration of a broader range of pedestal scenarios.

\section{Acknowledgments}

The first author would like to acknowledge useful discussions with M. Kotschenreuther, P. Valanju, and D. Ernst. Simulations were carried out using the HELIOS supercomputer system at the International Fusion Energy Research Centre, Aomori, Japan, under the Broader Approach collaboration between Euratom and Japan, implemented by Fusion for Energy and JAEA. Simulations were also performed at the Texas Advanced Computing Center (TACC) at The University of Texas at Austin. This work was supported by U.S. DOE Contract No. DE-FG02-04ER54742. 
[1] F. Wagner et al., Phys. Rev. Lett. 491408 (1982).

[2] E. J. Doyle et al., Nucl. Fusion 47, S18 (2007).

[3] J. W. Connor, Plasma Phys. Control. Fusion, 40, 531 (1998).

[4] G. T. A. Huysmans, Plasma Phys. Control. Fusion 47 B165 (2005).

[5] P. B. Snyder, N. Aiba, M. Beurskens, R. J. Groebner, L. D. Horton, A. E. Hubbard, J. W. Hughes, G. T. A. Huysmans, Y. Kamada, and A. Kirk, Nucl. Fusion, 49, 085035, (2009).

[6] K. H. Burrell, et al., Phys. Plasmas 12, 056121 (2005).

[7] L. J. Zheng, M. T. Kotschenreuther, and P. Valanju, Nucl. Fusion, 53, 063009 (2013).

[8] W. Suttrop et al., Plasma Phys. Control. Fusion 45, 1399 (2003).

[9] E. Hubbard, D. G. Whyte, R. M. Churchill, I. Cziegler, A. Dominguez, T. Golfinopoulos, J. W. Hughes, J. E. Rice, I. Bespamyatnov, M. J. Greenwald, N. Howard, B. Lipschultz, E. S. Marmar, M. L. Reinke, W. L. Rowan, and J. L. Terry, Phys. Plasmas, 18, 056115 (2011).

[10] D. G. Whyte, A. E. Hubbard, J. W. Hughes, B. Lipschultz, J. E. Rice, E. S. Marmar, M. Greenwald, I. Cziegler, A. Dominguez, T. Golfinopoulos, N. Howard, L. Lin, R. M. McDermott, M. Porkolab, M. L. Reinke, J. Terry, N. Tsujii, S. Wolfe, S. Wukitch, Y. Lin, and the Alcator C-Mod Team, Nucl. Fusion, 50, 105005 (2010).

[11] T. E. Evans, R. A. Moyer, J. G. Watkins, T. H. Osborne, P. R. Thomas, M. Becoulet, J. A. Boedo, E. J. Doyle, M. E. Fenstermacher, K. H. Finken, R. J. Groebner, M. Groth, J. H. Harris, G. L. Jackson, R. J. L. Haye, C. J. Lasnier, S. Masuzaki, N. Ohyabu, D. G. Pretty, H. Reimerdes, T. L. Rhodes, D. L. Rudakov, M. J. Schaffer, M. R. Wade, G. Wang, W. P. West, and L. Zeng, Nucl. Fusion, 45, 595 (2005).

[12] G. R. McKee, Z. Yan, C. Holland, R. J. Buttery, T. E. Evans, R. A. Moyer, S. Mordijck, R. Nazikian, T. L. Rhodes, O. Schmitz, and M. R. Wade, Nucl. Fusion, 53, 113011 (2013).

[13] J. D. Callen, R. J. Groebner, T. H. Osborne, J. M. Canik, L. W. Owen, A. Y. Pankin, T. Rafiq, T. D. Rognlien, and W. M. Stacey, Nucl. Fusion, 50, 064004 (2010).

[14] R. J. Groebner, C. S. Chang, J. W. Hughes, R. Maingi, P. B. Snyder, X. Q. Xu, J. A. Boedo, D. P. Boyle, J. D. Callen, J. M. Canik, I. Cziegler, E. M. Davis, A. Diallo, P. H. Diamond, J. D. Elder, D. P. Eldon, D. R. Ernst, D. P. Fulton, M. Landreman, A. W. Leonard, J. D. Lore, T. H. Osborne, A. Y. Pankin, S. E. Parker, T. L. Rhodes, S. P. Smith, A. C. Sontag, W. M. Stacey, J. Walk, W. Wan, E. H.-J. Wang, J. G. Watkins, A. E. White, D. G. Whyte, Z. Yan, E. A. Belli, B. D. Bray, J. Candy, R. M. Churchill, T. M. Deterly, E. J. Doyle, M. E. Fenstermacher, N. M. Ferraro, A. E. Hubbard, I. Joseph, J. E. Kinsey, B. LaBombard, C. J. Lasnier, Z. Lin, B. L. Lipschultz, C. Liu, Y. Ma, G. R. McKee, D. M. Ponce, J. C. Rost, L. Schmitz, G. M. Staebler, L. E. Sugiyama, J. L. Terry, M. V. Umansky, R. E. Waltz, S. M. Wolfe, L. Zeng, and S. J. Zweben, Nucl. Fusion, 53, 093024 (2013).

[15] F. L. Hinton and R. D. Hazeltine, Rev. Mod. Phys., 48, 239 (1976).

[16] J. D. Callen, Phys. Plasmas 14, 040701 (2007).

[17] S. P. Smith, J. D. Callen, R. J. Groebner, T. H. Osborne, A. W. Leonard, D. Eldon, B. D. Bray, and the D.-D. Team, Nucl. Fusion, 52, 114016 (2012).

[18] W. Wan, S. E. Parker, Y. Chen, Z. Yan, R. J. Groebner, and P. B. Snyder, Phys. Rev. Lett., 109, 185004, (2012).

[19] E. Wang, X. Xu, J. Candy, R. J. Groebner, P. B. Snyder, Y. Chen,
S. E. Parker, W. Wan, G. Lu, and J. Q. Dong, Nucl. Fusion, 52, 103015 (2012).

[20] W. Wan, S. E. Parker, Y. Chen, R. J. Groebner, Z. Yan, A. Y. Pankin, and S. E. Kruger, Phys. Plasmas, 20, 055902 (2013).

[21] D. Dickinson, C. M. Roach, S. Saarelma, R. Scannell, A. Kirk, and H. R. Wilson, Phys. Rev. Lett., 108, 135002 (2012).

[22] F. Jenko, W. Dorland, M. Kotschenreuther, and B. N. Rogers, Phys. Plasmas, 7, 1904 (2000).

[23] E. Wolfrum, A. Burckhart, R. Fischer, N. Hicks, C. Konz, B. Kurzan, B. Langer, T. Pütterich, H. Zohm, and the A. U. Team, Plasma Phys. Control. Fusion, 51, 124057 (2009).

[24] A. Burckhart, E. Wolfrum, R. Fischer, K. Lackner, H. Zohm, and the A. U. Team, Plasma Phys. Control. Fusion, 52, 105010 (2010).

[25] S. K. Rathgeber, L. Barrera, T. Eich, R. Fischer, B. Nold, W. Suttrop, M. Willensdorfer, and E. Wolfrum, Plasma Phys. Control. Fusion, 55, 025004 (2013).

[26] E. Viezzer, T. Pütterich, G. D. Conway, R. Dux, T. Happel, J. C. Fuchs, R. M. McDermott, F. Ryter, B. Sieglin, W. Suttrop, M. Willensdorfer, E. Wolfrum, and the ASDEX Upgrade Team, Nucl. Fusion, 53, 053005 (2013).

[27] P. J. McCarthy, Phys. Plasmas, 6, 3554 (1999).

[28] P. J. McCarthy and the ASDEX Upgrade Team, Plasma Phys. Control. Fusion, 54, 015010 (2012).

[29] M. G. Dunne, P. J. McCarthy, E. Wolfrum, R. Fischer, L. Giannone, A. Burckhart, and the ASDEX Upgrade Team, Nucl. Fusion, 52, 123014 (2012).

[30] E. Viezzer, T. Pütterich, R. M. McDermott, G. D. Conway, M. Cavedon, M. G. Dunne, R. Dux, E. Wolfrum, and the ASDEX Upgrade Team, Plasma Phys. Control. Fusion, 56, 075018 (2014).

[31] P. W. Terry, Reviews of Modern Physics, 72, 109 (2000).

[32] T. S. Hahm and K. H. Burrell, Phys. Plasmas, 2, 1648 (1995).

[33] M. A. Beer, S. C. Cowley, and G. W. Hammett, Phys. Plasmas 2, 2687 (1995).

[34] M. J. Pueschel, T. Dannert, and F. Jenko, Computer Physics Communications 181, 1428 (2010).

[35] H. Nordman, J. Weiland, and A. Jarmén, Nucl. Fusion 30, 983 (1990).

[36] T. Dannert and F. Jenko, Phys. Plasmas, 12, 072309 (2005).

[37] D. R. Ernst, J. Lang, W. M. Nevins, M. Hoffman, Y. Chen, W. Dorland, and S. Parker, Phys. Plasmas, 16, 055906 (2009).

[38] D. R. Ernst et al., Bull. Am. Phys. Soc. 50 (8) (2005) 235.

[39] D. P. Fulton, Z. Lin, I. Holod, and Y. Xiao, Phys. Plasmas 21, 042110 (2014).

[40] J. F. Drake and Y. C. Lee, Phys. Rev. Lett. 39, 453 (1977).

[41] D. Dickinson, C. M. Roach, S. Saarelma, R. Scannell, A. Kirk, and H. R. Wilson, Plasma Phys. Control. Fusion, 55, 074006 (2013).

[42] S. Saarelma, M. N. A. Beurskens, D. Dickinson, L. Frassinetti, M. J. Leyland, C. M. Roach, and E.-J. Contributors, Nucl. Fusion, 53, 123012 (2013).

[43] Z. Yan, G. R. McKee, R. J. Groebner, P. B. Snyder, T. H. Osborne, and K. H. Burrell, Phys. Rev. Lett., 107, 055004 (2011).

[44] Z. Yan, G. R. McKee, R. J. Groebner, P. B. Snyder, T. H. Osborne, M. N. Beurskens, and K. H. Burrell, Phys. Plasmas, 18, 056117 (2011).

[45] F. Jenko, W. Dorland, and G. W. Hammett, Phys. Plasmas, 8, 4096 (2001).

[46] W. M. Nevins, J. Candy, S. Cowley, T. Dannert, A. Dimits, W. Dorland, C. Estrada-Mila, G. W. Hammett, F. Jenko, M. 
J. Pueschel, and D. E. Shumaker, Phys. Plasmas, 13, 122306 (2006).

[47] D. Told, F. Jenko, P. Xanthopoulos, L. D. Horton, E. Wolfrum, and ASDEX Upgrade Team, Phys. Plasmas, 15, 102306 (2008).

[48] F. Jenko, D. Told, P. Xanthopoulos, F. Merz, and L. D. Horton, Phys. Plasmas, 16, 055901 (2009).

[49] D. Told, PhD Thesis, Universität Ulm (2012).

[50] P. B. Snyder, R. J. Groebner, A. W. Leonard, T. H. Osborne, and H. R. Wilson, Phys. Plasmas 16, 056118 (2009).

[51] M. J. Pueschel, M. Kammerer, F. Jenko, Phys. Plasmas 15, 102310 (2008).

[52] M. J. Pueschel and F. Jenko, Phys. Plasmas 17, 062307 (2010).

[53] M. Kotschenreuther, Phys. Fluids, 29, 2898 (1986).

[54] W. M. Stacey and R. J. Groebner, Phys. Plasmas, 16, 102504 (2009).

[55] M. Willensdorfer, E. Fable, E. Wolfrum, L. Aho-Mantila, F. Aumayr, R. Fischer, F. Reimold, F. Ryter, and the ASDEX Upgrad Team, Nucl. Fusion, 53, 093020 (2013).

[56] M. A. Mahdavi, R. Maingi, R. J. Groebner, A. W. Leonard, T. H. Osborne, and G. Porter, Phys. of Plasmas, 10, 3984 (2003).

[57] A. S. Kukushkin, H. D. Pacher, A. Loarte, V. Komarov, V. Kotov, M. Merola, G. W. Pacher, and D. Reiter, Nucl. Fusion, 49, 075008 (2009).

[58] H. Doerk, F. Jenko, M. J. Pueschel, and D. R. Hatch, Phys. Rev. Lett., 106, 155003 (2011).
[59] W. Guttenfelder, J. Candy, S. M. Kaye, W. M. Nevins, E. Wang, R. E. Bell, G. W. Hammett, B. P. LeBlanc, D. R. Mikkelsen, and H. Yuh, Phys. Rev. Lett. 106, 155004 (2011).

[60] D. R. Hatch, P. W. Terry, W. M. Nevins, and W. Dorland, Phys. Plasmas, 16, 022311 (2009).

[61] P. W. Terry, D. A. Baver, and S. Gupta, Phys. Plasmas 13, 022307 (2006).

[62] D. R. Hatch, P. W. Terry, F. Jenko, F. Merz, and W. M. Nevins, Phys. Rev. Lett. 106, 115003 (2011).

[63] D. R. Hatch et al., Phys. Plasmas 18, 055706 (2011).

[64] J. Citrin, C. Bourdelle, P. Cottier, D. F. Escande, Ö. D. Gürcan, D. R. Hatch, G. M. D. Hogeweij, F. Jenko, and M. J. Pueschel, Phys. Plasmas, 19, 062305 (2012).

[65] B. D. Scott, New J. Phys., 4, 52 (2002).

[66] P. H. Diamond, S.-I. Itoh, K. Itoh, and T. S. Hahm, Plasma Phys. Controlled Fusion 47 (2005) R35.

[67] M. J. Pueschel, P. W. Terry, F. Jenko, D. R. Hatch, W. M. Nevins, T. Görler, and D. Told, Phys. Rev. Lett., 110, 155005 (2013).

[68] M. J. Pueschel, D. R. Hatch, T. Görler, W. M. Nevins, F. Jenko, P. W. Terry, and D. Told, Phys. Plasmas, 20, 102301 (2013).

[69] M. J. Pueschel, P. W. Terry, and D. R. Hatch, Phys. Plasmas, 21, 055901 (2014). 